\section{Characterization of the human inflammatory response to spinal cord injury}

Spinal cord injury (SCl) induces an inflammatory response that initially causes secondary tissue damage, but which is also thought to contribute to tissue repair later after injury. Many animal studies have been conducted to establish the time course of tissue entry for different inflammatory cells during this response, and in a recent study researchers characterized this time course in human spinal cords.

Fleming et al. investigated the postmortem spinal cords of patients who sustained $\mathrm{SCl}$ and either died immediately from their injury, or survived for up to 1 year afterwards. Crosssectional samples were divided into distinct areas that were assigned ratings regarding their overall damage. The time course of entry into tissue for the different inflammatory cells was assessed using immunohistochemistry and quantitative analysis.

Neutrophils were the first cell type to enter the region of tissue damage; these cells were present by $4 \mathrm{~h}$ after injury, reached their maximum number $1-3$ days following injury, and were still present 10 days after injury. Activated microglia were also present $1-3$ days after $\mathrm{SCl}$, and the presence of both cell types correlated with areas of increased tissue damage. By 510 days after $\mathrm{SCl}$, macrophages and activated microglia were the main inflammatory cells to be detected in damaged tissue.

The authors conclude that the inflammatory events occurring after $\mathrm{SCl}$ in humans are similar to those already documented in rodent models, and they note that monoclonal antibody treatment to halt early entry of neutrophils into damaged tissue-already shown to improve neurological outcome in animals-could be used as a neuroprotective therapy in humans.

Original article Fleming JC et al. (2006) The cellular inflammatory response in human spinal cords after injury. Brain 129: 3249-3269

\section{New evidence of serotonergic dysfunction in sudden infant death syndrome}

Sudden infant death syndrome (SIDS) is a major cause of postneonatal infant death, but despite considerable research into the condition the underlying biological mechanisms remain unclear. Abnormal binding patterns of the 5-hydroxytryptamine (5-HT; serotonin) receptors have previously been reported in cases of SIDS, and in a recent postmortem study of SIDS infants, researchers have found new evidence of cellular alterations within the serotonergic system of the medulla oblongata.

Paterson et al. obtained frozen medullae from 31 infants who had died from SIDS and from 10 infants who had died acutely from other causes (controls). The number and density of serotonergic neurons within the medullae were determined using immunocytochemistry, and the binding densities of the $5-\mathrm{HT}_{1 \mathrm{~A}}$ receptor and 5-HT transporter were assessed with autoradiography. The researchers also investigated potential correlations between their results and known risk factors for SIDS.

In SIDS cases, regions of the medulla involved in homeostatic function had significantly higher serotonergic neuron counts and densities than those of controls $(P<0.001$ for both), and significantly lower $5-\mathrm{HT}_{1 \mathrm{~A}}$ receptor binding densities $(P \leq 0.01)$. In the raphé obscurus, male SIDS cases had significantly lower $5-\mathrm{HT}_{1 \mathrm{~A}}$ receptor binding densities than female SIDS cases $(P=0.04)$.

The authors conclude that the medullary serotonergic abnormalities in SIDS cases are more extensive than have previously been reported, and that their results generate new hypotheses regarding 5-HT-related brainstem pathology in early sudden death. They also suggest that the lower levels of $5-\mathrm{HT}_{1 \mathrm{~A}}$ receptor binding density in male SIDS cases than in female cases might explain the particular vulnerability of males to this condition.

Original article Paterson DS et al. (2006) Multiple serotonergic brainstem abnormalities in sudden infant death syndrome. JAMA 296: 2124-2132

\section{Spreading depolarizations are indicators of brain damage after subarachnoid hemorrhage}

Progressive ischemic damage in animals is characterized by spreading mass depolarizations of neurons, detected as spreading negative slow voltage variation. It is unclear, however, whether such spreading depolarizations occur in ischemic stroke in humans. Dreier et al. investigated the incidence of spreading 